\title{
Evolução da obesidade e doenças crônicas não transmissíveis nas populações das capitais do Brasil entre 2006 e 2018
}

\author{
Eduardo Botti Abbade ${ }^{1}$ D
}

\begin{abstract}
RESUMO
Modelo do estudo: Estudo descritivo transversal. Objetivo: O objetivo deste estudo foi analisar a evolução da prevalência de sobrepeso, obesidade e doenças crônicas não transmissíveis (DCNT), e sua relação com idade e anos de estudo, nas capitais brasileiras. Método: Foram analisados dados oriundos da Pesquisa VIGITEL prioritariamente de 2006 e 2018 referentes à 12 variáveis, através de procedimentos estatísticos descritivos, análises de frequência e diagramas de dispersão com inserção das curvas de tendência e coeficientes de determinação. Resultados: Os resultados evidenciam um aumento expressivo do IMC médio e das prevalências DCNT nas populações das capitais do Brasil, embora a autopercepção do estado geral de saúde apresente alteração inexpressiva. O IMC médio da população é maior na faixa etária entre 45 e 65 anos, e as prevalências de diabetes, pressão alta e dislipidemia apresentam um crescimento acentuado partir dos 40 anos, atingindo o ápice na faixa etária entre 70 e 80 anos. Quanto mais anos de estudos possui a população, menor são as prevalências de obesidade e DCNT. Conclusão: Iniciativas, tanto públicas como privadas, de redução dos fatores de risco que potencializam o aumento da obesidade e de DCNT são necessárias. Ademais, o aumento do nível educacional de uma população tem potencial de promover melhoria significativa no quadro de saúde pública, reduzindo os gastos em saúde e melhorando a qualidade de vida populacional.
\end{abstract}

Palavras-chave: Obesidade; Doenças não Transmissíveis; Diabetes; Doenças Cardiovasculares; Saúde Pública.

\section{INTRODUÇÃO}

A obesidade é doença multifatorial, crônica e progressiva, que afeta parcelas consideráveis da população mundial e brasileira ${ }^{1-4}$. De acordo com a Organização Mundial da Saúde, mais de 1,9 bilhão de adultos (pessoas com mais de 18 anos) estava acima do peso (39\%) em 2014, e mais de 600 milhões eram considerados obesos $(13 \%)^{5}$. No Brasil, a elevada prevalência da obesidade observada na população é fator preocupante, uma vez que, segundo dados do Ministério da Saúde, em 2013 cerca de $30 \%$ das crianças do país apresentavam sobrepeso ou obesidade. Dados mais atualizados disponibilizados através do VIGITEL 2017, amplo estudo de vigilância de fatores de risco e proteção para doenças crônicas por inquérito telefônico feito anualmente pelo Ministério da Saúde, revelou que cerca de 18,9\% da população estava diagnosticada como obesa e $54 \%$ estava com sobrepeso ${ }^{6}$.
A obesidade coletiva, fenômeno observado em diversas populações em todo o mundo, tem graves implicações para a saúde pública, além de implicações sociais, econômicas e ambientais. Considerando aspectos associados à saúde do indivíduo e à saúde pública, estudos anteriores mostraram que a prevalência de obesidade tem potencial de elevar o risco de morbidade e mortalidade por diversas DCNT (Doenças Crônicas Não Transmissíveis), como diabetes mellitus, doença cardiovascular, doenças respiratórias crônicas, depressão e câncer ${ }^{7-9}$, resultando em um potencial aumento de gastos no âmbito da saúde pública ${ }^{10-12}$. Nos Estados Unidos, estudo anterior demonstrou que os custos médicos associados à obesidade chegam a US $\$ 209,7$ bilhões e que a redução efetiva do peso da população poderia gerar redução de custo na ordem de US\$ 610 bilhões em 20 anos $^{13}$. No Brasil, estudo anterior evidenciou que em 2017 foram financiados pelo SUS 10.840 procedimentos de gastroplastia totalizando cerca de R\$ 69 milhões $^{14}$.

Universidade Federal de Santa Maria. Departamento de Ciências Administrativas, (UFSM), Santa Maria (RS), Brasil. 
As DCNT representam um problema grave à saúde pública mundialmente e no Brasil. De fato, as DCNT são as maiores responsáveis por mortes no mundo, sendo as doenças cardiovasculares a principal dentre elas ${ }^{15}$. As principais DCNT (doenças cardiovasculares, câncer, diabetes, enfermidades respiratórias crônicas e doenças neuropsiquiátricas) foram associadas a cerca de $74 \%$ das mortes no Brasil em 2016, sendo a taxa de risco para morte prematura decorrente de DCNT para pessoas com idade entre 30 e 70 anos estimada em 17\%. Além da morte prematura, as DCNT também implicam em grande perda de qualidade de vida e custos econômicos e sociais ${ }^{16}$.

Considerando a urgência do tema, este estudo objetivou analisar a evolução da prevalência de sobrepeso, obesidade (níveis I, II e III) e DCNT (diabetes, pressão alta, dislipidemias e doenças cardiovasculares), e sua relação com idade e anos de estudo, nas capitais brasileiras.

\section{MÉTODOS}

Este estudo analisa dados do sistema de Vigilância de Fatores de Risco para doenças crônicas não transmissíveis (DCNT) do Ministério da
Saúde (VIGITEL) nos anos de 2006 a 2018. Essa pesquisa sistemática conduzida pelo Ministério da Saúde foi iniciada em 2006 e é realizada anualmente pelo governo brasileiro, gerando dados anuais sobre populações adultas ( $\geq 18$ anos) residentes nas 26 capitais brasileiras e no Distrito Federal. O método adotado pelo sistema VIGITEL é bastante robusto, sendo estabelecido que o tamanho mínimo da amostra em cada uma das capitais deve ser de pelo menos 2.000 entrevistas telefônicas, para estimar a frequência das variáveis investigadas com um coeficiente de confiança de $95 \%$ e erro amostral de $2 \%{ }^{17}$.

Para os fins específicos desta investigação, foram compilados dados de quatro edições da VIGITEL (2006, 2008, 2016 e 2018), referentes à 12 variáveis chaves que estão detalhadas no Quadro 1 a seguir, as quais foram autorreferidas.

A partir da base de dados construída para o estudo, foi calculado o IMC para cada um dos registros, utilizando a fórmula padrão do IMC = [peso]/ [altura] ${ }^{2}$. Na sequência, os valores de IMC obtidos foram classificados de acordo com as categorias previstas pela Organização Mundial da Saúde (OMS), sendo: (1) normal [IMC <25]; (2) sobrepeso [IMC $\geq 25]$; (3) obesidade I [IMC $\geq 30$ ]; (4) obesidade II [IMC $\geq 35]$; e (5) obesidade III $[\text { IMC } \geq 40]^{18}$.

\section{Quadro 1}

Variáveis da VIGITEL analisadas no estudo

\begin{tabular}{|c|c|c|c|}
\hline Código & Variável & Descrição da questão & Anos analisados \\
\hline cidade & Capital do BR & Cidade/Estado & 2006 e 2018 \\
\hline q6 & Idade (anos) & Qual sua idade? (Só aceita $\geq 18$ anos e $<150$ anos) & 2006 e 2018 \\
\hline q7 & Sexo & Sexo $[1=$ masculino; 2 = feminino $]$ & 2006 e 2018 \\
\hline anoescol & Anos de Estudo & $\begin{array}{l}\text { Calculada tendo como base a questão q8 (Até que série e grau } \\
\text { o(a) Sr.(a) estudou?). }\end{array}$ & 2006 e 2018 \\
\hline q9 & Peso $(\mathrm{kg})$ & $\begin{array}{l}\text { O(a) Sr.(a) sabe seu peso (mesmo que seja valor aproximado)? } \\
\text { (Só aceita } \geq 30 \mathrm{~kg} \mathrm{e}<300 \mathrm{~kg} \text { ) }\end{array}$ & 2006 e 2018 \\
\hline q11 & Altura $(\mathrm{cm})$ & O(a) Sr.(a) sabe sua altura? (Só aceita $\geq 1,20 \mathrm{~m} \mathrm{e}<2,20 \mathrm{~m}$ ) & 2006 e 2018 \\
\hline $\mathrm{q} 14$ & Gravidez & A Sra. está grávida no momento? [1 = sim; 2 = não] & 2006 e 2018 \\
\hline $\mathrm{q} 74$ & $\begin{array}{l}\text { Percepção } \\
\text { do Estado de } \\
\text { Saúde }\end{array}$ & $\begin{array}{l}\text { O(a) Sr.(a) classificaria seu estado de saúde como: }[1=\text { muito } \\
\text { bom; } 2=\text { bom; } 3=\text { regular; } 4=\text { ruim; } 5=\text { muito ruim; } 777= \\
\text { não sabe; } 888=\text { não quis informar }]\end{array}$ & 2006 e 2018 \\
\hline q75 & Pressão Alta & $\begin{array}{l}\text { Algum médico já lhe disse que o(a) Sr.(a) tem pressão alta? [1 = } \\
\text { sim; } 2 \text { = não] }\end{array}$ & 2006 e 2018 \\
\hline q76 & Diabetes & $\begin{array}{l}\text { Algum médico já lhe disse que o(a) Sr.(a) tem diabetes? }[1=\text { sim; } \\
2=\text { não }]\end{array}$ & 2006 e 2018 \\
\hline $\mathrm{q} 77$ & $\begin{array}{l}\text { Infarto, } \\
\text { derrame, AVC }\end{array}$ & $\begin{array}{l}\text { Algum médico já lhe disse que o(a) Sr.(a) teve infarto, derrame } \\
\text { ou acidente vascular cerebral (AVC)? }[1=\operatorname{sim} ; 2=\text { não }]\end{array}$ & 2008 \\
\hline $\mathrm{q} 78$ & Dislipidemia & $\begin{array}{l}\text { Algum médico já Ihe disse que o(a) Sr.(a) tem colesterol ou } \\
\text { triglicérides elevado? }[1=\operatorname{sim} ; 2=\text { não }]\end{array}$ & 2006 e 2016 \\
\hline
\end{tabular}


Os dados foram analisados utilizando procedimentos estatísticos descritivos e análises de frequência. Especificamente para a análise da relação entre a idade, anos de estudo e indicadores de prevalência de obesidade e DCNT autorreferidas foram utilizados diagramas de dispersão com inserção das equações das curvas de tendência e seus respectivos coeficientes de determinação $\left(R^{2}\right)$. As seguintes variáveis foram consideradas como sendo variáveis dependentes, impactadas pela idade e pela quantidade de anos de estudo: (1) IMC médio; (2) prevalência de obesidade I; (3) prevalência de obesidade II; (4) prevalência de diabetes; (5) prevalência de pressão alta; (6) prevalência de dislipidemia; (7) prevalência de infarto, derrame e AVC; e (8) autoavaliação do estado geral de saúde.

O estudo procedeu com a análise comparativa dos dados do VIGITEL para os anos de 2006 e 2018. Especificamente para a dislipidemia, a comparação é feita entre os anos de 2006 e 2016 já que este foi o último ano com dados disponíveis para essa variável. Para o cálculo do IMC médio foram desconsideradas as mulheres que manifestaram situação de gravidez no momento da coleta de dados.

Cabe destacar que o sistema VIGITEL foi aprovado pelo Comitê Nacional de Ética em Pesquisa com Seres Humanos (CONEP) do Ministério da Saúde do Brasil e, por se tratar de uma entrevista por telefone, foi obtido o consentimento verbal, ao invés do termo de consentimento livre e esclarecido.

\section{RESULTADOS E DISCUSSÃO}

A Tabela 1 apresenta a análise da evolução do IMC médio, percepção geral do estado de saúde, prevalência de pressão alta, diabetes e dislipidemia.

\section{Tabela 1}

Avaliação dos indicadores de saúde e DCNT nas Capitais do Brasil

\begin{tabular}{|c|c|c|c|c|c|c|c|c|c|c|}
\hline & \multicolumn{2}{|c|}{ IMC Médioa } & \multicolumn{2}{|c|}{$\begin{array}{l}\text { Severidade do } \\
\text { Estado de Saúde }\end{array}$} & \multicolumn{2}{|c|}{ Pressão Altac } & \multicolumn{2}{|c|}{ Diabetes $^{c}$} & \multicolumn{2}{|c|}{ Dislipidemiac $^{c}$} \\
\hline & 2006 & 2018 & 2006 & 2018 & 2006 & 2018 & 2006 & 2018 & 2006 & 2016d \\
\hline BRASILe & 24,40 & 26,41 & 2,25 & 2,25 & $23,83 \%$ & $34,98 \%$ & $5,57 \%$ & $11,26 \%$ & $20,35 \%$ & $30,47 \%$ \\
\hline 1- Aracajú & 24,32 & 26,33 & 2,28 & 2,29 & $24,37 \%$ & $36,23 \%$ & $5,47 \%$ & $11,59 \%$ & $27,16 \%$ & $37,57 \%$ \\
\hline 2- Belém & 24,39 & 26,47 & 2,35 & 2,27 & $20,79 \%$ & $29,30 \%$ & $5,40 \%$ & $9,85 \%$ & $22,29 \%$ & $33,28 \%$ \\
\hline 3- Belo Horizonte & 23,84 & 26,24 & 2,10 & 2,14 & $25,69 \%$ & $40,01 \%$ & $4,67 \%$ & $12,36 \%$ & $19,14 \%$ & $29,49 \%$ \\
\hline 4- Boa Vista & 24,50 & 26,35 & 2,34 & 2,26 & $21,18 \%$ & $26,17 \%$ & $4,86 \%$ & $8,33 \%$ & $15,70 \%$ & $23,64 \%$ \\
\hline 5- Campo Grande & 24,66 & 26,88 & 2,15 & 2,20 & $26,22 \%$ & $39,85 \%$ & $5,16 \%$ & $12,80 \%$ & $17,94 \%$ & $31,33 \%$ \\
\hline 6- Cuiabá & 24,83 & 27,01 & 2,22 & 2,23 & $23,88 \%$ & $33,53 \%$ & $5,36 \%$ & $10,55 \%$ & $14,17 \%$ & $24,95 \%$ \\
\hline 7- Curitiba & 24,91 & 26,35 & 2,13 & 2,17 & 24,4 & 35 & $6,36 \%$ & 12 & $\%$ & $7 \%$ \\
\hline 8- Florianópolis & 24,30 & 26,28 & 2,08 & 2,11 & & & 5,1 & 11, & 22, & $2 \%$ \\
\hline 9- Fortaleza & 24,20 & 26,73 & 2,22 & 2,28 & $22,51 \%$ & $33,25 \%$ & $5,72 \%$ & $14,94 \%$ & $18,44 \%$ & $30,09 \%$ \\
\hline 10- Goiânia & 24,20 & 26,19 & 2,23 & 2,24 & $24,70 \%$ & $37,57 \%$ & $6,18 \%$ & $11,33 \%$ & $20,08 \%$ & $29,72 \%$ \\
\hline 11- João Pess & 24,50 & 26,28 & 2,28 & 2,29 & 27,8 & & 6,3 & 11 & $\%$ & $3 \%$ \\
\hline 12- Macapá & 24,85 & 26,51 & 2,37 & 2,27 & $21,99 \%$ & & $4,92 \%$ & $\%$ & $17,82 \%$ & $7 \%$ \\
\hline 13- Maceió & 24,09 & 26,15 & 2,37 & 2,36 & $26,27 \%$ & $38,57 \%$ & $7,19 \%$ & $13,75 \%$ & $22,93 \%$ & $34,56 \%$ \\
\hline 14- Manaus & 24,96 & 26,97 & 2,32 & 2,29 & $21,73 \%$ & $30,36 \%$ & $5,20 \%$ & $9,06 \%$ & $20,49 \%$ & $31,58 \%$ \\
\hline 15- Natal & 24,72 & 26,33 & 2,28 & 2,27 & $25,51 \%$ & $35,64 \%$ & $5,77 \%$ & $11,57 \%$ & $25,14 \%$ & $34,89 \%$ \\
\hline 16- Palmas & 23,97 & 25,97 & 2,18 & 2,14 & $15,29 \%$ & 25 , & $3,50 \%$ & $7,23 \%$ & $2 \%$ & $27,19 \%$ \\
\hline 17- Porto Alegre & 24,98 & 26,60 & 2,09 & 2,14 & $25,31 \%$ & $39,56 \%$ & $5,12 \%$ & $13,43 \%$ & $22,90 \%$ & $29,88 \%$ \\
\hline 18- Porto Velho & 24,64 & 26,63 & 2,27 & 2,25 & $21,53 \%$ & $29,79 \%$ & $4,95 \%$ & $9,57 \%$ & $18,07 \%$ & $25,62 \%$ \\
\hline 19- Recife & 24,61 & 26,45 & 2,31 & 2,38 & $29,31 \%$ & $39,25 \%$ & $6,13 \%$ & $11,67 \%$ & $21,53 \%$ & $31,17 \%$ \\
\hline 20- Rio Branco & 24,65 & 26,86 & 2,40 & 2,29 & $24,78 \%$ & $35,80 \%$ & $4,35 \%$ & $9,17 \%$ & $17,84 \%$ & $25,75 \%$ \\
\hline 21- Rio de Janeiro & 24,68 & 26,70 & 2,26 & 2,31 & $29,93 \%$ & $44,13 \%$ & $7,03 \%$ & $14,21 \%$ & $22,79 \%$ & $28,34 \%$ \\
\hline 22- Salvador & 24,06 & 26,29 & 2,31 & 2,36 & $27,25 \%$ & $36,65 \%$ & $5,84 \%$ & $10,59 \%$ & $22,23 \%$ & $34,50 \%$ \\
\hline 23- São Luís & 23,27 & 25,73 & 2,37 & 2,31 & $20,08 \%$ & $28,99 \%$ & $6,03 \%$ & $9,73 \%$ & $19,13 \%$ & $29,27 \%$ \\
\hline 24- São Paulo & 24,78 & 26,69 & 2,21 & 2,21 & $24,77 \%$ & $35,38 \%$ & $7,50 \%$ & $11,84 \%$ & $16,68 \%$ & $26,11 \%$ \\
\hline 25- Teresina & 23,59 & 25,95 & 2,37 & 2,30 & $20,41 \%$ & $32,72 \%$ & $4,78 \%$ & $8,73 \%$ & $18,96 \%$ & $30,68 \%$ \\
\hline 26- Vitória & 24,03 & 26,15 & 2,19 & 2,13 & $26,10 \%$ & $37,61 \%$ & $5,79 \%$ & $12,19 \%$ & $22,24 \%$ & $32,45 \%$ \\
\hline 27- Distrito Federal & 24,46 & 26,13 & 2,10 & 2,23 & $21,19 \%$ & $35,18 \%$ & $5,69 \%$ & $12,09 \%$ & $21,75 \%$ & $31,74 \%$ \\
\hline
\end{tabular}

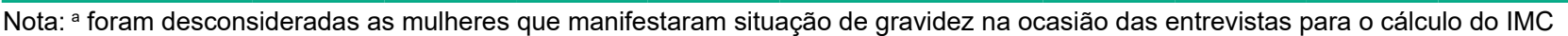
médio; b Variável baseada na autopercepção de saúde avaliada através da escala de 5 pontos indo de 1 (muito bom) a 5 (muito ruim); ${ }^{c}$ variável mensurada através da escala dicotômica (1=sim; 2=não); ${ }^{d}$ Foi utilizado o ano de 2016, pois esse foi o último ano disponível com dados para esta variável; ${ }^{c}$ Média Geral considerando as capitais do Brasil. 
Os resultados mostram um aumento expressivo do IMC médio na população residente nas capitais do Brasil, indo de 24,4 em 2006 para 26,41 em 2018. Observa-se ainda que o IMC médio aumentou cerca de 2 pontos em todas as capitais do país, e que Cuiabá, Manaus e Campo Grande são as capitais com os maiores valores de IMC médio na população em 2018, com 27,01, 26,97 e 26,88, respectivamente. Observa-se ainda que as prevalências de pressão alta, diabetes e dislipidemia aumentaram expressivamente nas capitais do Brasil no período analisado. A população das capitais do Brasil com pressão alta subiu de 23,83\% em 2006 para $34,98 \%$ em 2018 , a prevalência de diabetes subiu de 5,57\% em 2006 para 11,26\% em 2018, e a parcela populacional com dislipidemia subiu de 20,35\% em 2006 para 30,47\% em 2016. Embora as prevalências de pressão alta, diabetes e dislipidemia tenha aumentado expressivamente nas capitais do Brasil, a autopercepção do estado de saúde da população apresentou alteração inexpressiva, o que sugere que, embora as DCNT autorreferidas estejam aumentando drasticamente nos últimos anos, a população do país não avalia que tal aumento seja indicador de piora no seu quadro geral de saúde.

Com relação à prevalência de diabetes, doença responsável por inúmeras complicações de saúde e elevados custos, os resultados mostram que Fortaleza, Rio de Janeiro e Maceió apresentaram parcelas bastante expressivas em 2018 desta DCNT, sendo $14,94 \%, 14,21 \%$ e $13,75 \%$ da população respectivamente. Rio de Janeiro, Belo Horizonte, Campo Grande, Porto Alegre e Recife também apresentam parcelas expressivas da população com pressão alta em 2018. Também é observado que Aracajú, Natal e Maceió apresentam parcelas elevadas da população com dislipidemia em 2016.

Na sequência, a Tabela 2 apresenta a evolução da prevalência de sobrepeso e dos três níveis de obesidade observados nas populações das capitais do Brasil para os anos de 2006 e 2018. Cabe salientar que diagnósticos com base em IMC superiores também são contabilizados nos diagnósticos de IMC inferior, ou seja, por exemplo, a população com obesidade nível III também é contabilizada na população com obesidade níveis II, I e sobrepeso.

As prevalências de sobrepeso e obesidade, em seus três níveis, apresentam expressivo aumento no período considerado no estudo. De fato, a parcela da população residente nas capitais do Brasil em situação de sobrepeso passou de $39,06 \%$ em 2006 para 51,93\% em 2018, o que indica que mais da metade da população das capitais brasileiras está com sobrepeso. As prevalências dos três níveis de obesidade também apresentam elevado aumento, uma vez que a prevalência de obesidade I passou de 10,73\% em 2006 para 17,87\% em 2018, a prevalência de obesidade II passou de $2,63 \%$ em 2006 para 4,66\% em 2018 e a prevalência de obesidade III passou de 0,73\% em 2006 para 1,24\% em 2018, sugerindo que a obesidade coletiva está aumentando de forma alarmante no Brasil.

\section{Tabela 2}

Prevalência de sobrepeso e tipos de obesidade nas capitais do Brasil (2006 e 2018)

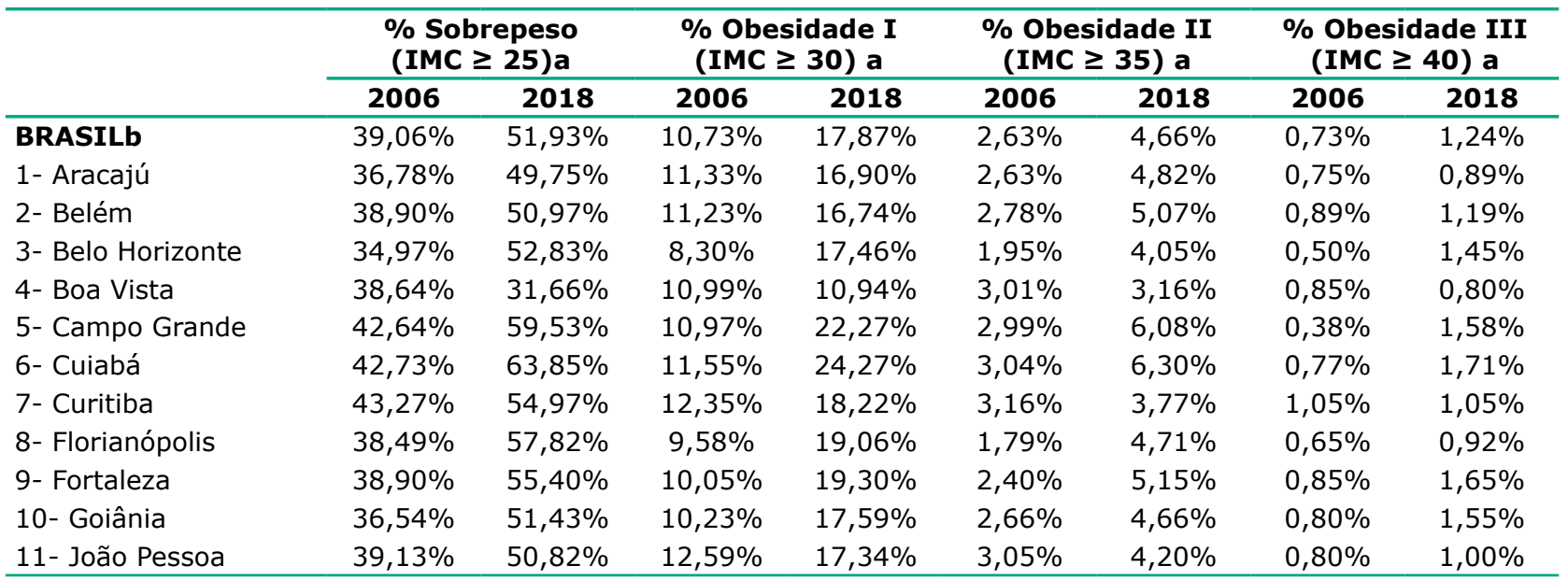




\begin{tabular}{|c|c|c|c|c|c|c|c|c|}
\hline & \multicolumn{2}{|c|}{$\begin{array}{l}\% \text { Sobrepeso } \\
(\text { IMC } \geq 25) a\end{array}$} & \multicolumn{2}{|c|}{$\begin{array}{l}\% \text { Obesidade I } \\
(I M C \geq 30) \text { a }\end{array}$} & \multicolumn{2}{|c|}{$\begin{array}{l}\% \text { Obesidade II } \\
(\text { IMC } \geq 35) \text { a }\end{array}$} & \multicolumn{2}{|c|}{$\begin{array}{l}\% \text { Obesidade III } \\
(\text { IMC } \geq 40) \text { a }\end{array}$} \\
\hline & 2006 & 2018 & 2006 & 2018 & 2006 & 2018 & 2006 & 2018 \\
\hline 12- Macapá & $41,27 \%$ & $38,52 \%$ & $12,41 \%$ & $13,59 \%$ & $3,43 \%$ & $4,44 \%$ & $1,01 \%$ & $1,07 \%$ \\
\hline 13- Maceió & $36,47 \%$ & $49,25 \%$ & $10,71 \%$ & $15,79 \%$ & $2,67 \%$ & $4,38 \%$ & $0,45 \%$ & $0,91 \%$ \\
\hline 14- Manaus & $42,03 \%$ & $46,52 \%$ & $13,02 \%$ & $17,61 \%$ & $3,28 \%$ & $4,64 \%$ & $1,01 \%$ & $1,46 \%$ \\
\hline 15- Natal & $40,54 \%$ & $52,28 \%$ & $11,69 \%$ & $18,72 \%$ & $3,01 \%$ & $3,66 \%$ & $0,75 \%$ & $0,80 \%$ \\
\hline 16- Palmas & $35,45 \%$ & $52,79 \%$ & $8,62 \%$ & $16,13 \%$ & $1,72 \%$ & $3,65 \%$ & $0,61 \%$ & $0,61 \%$ \\
\hline 17- Porto Alegre & $45,35 \%$ & $63,56 \%$ & $12,05 \%$ & $21,93 \%$ & $3,31 \%$ & $5,65 \%$ & $1,09 \%$ & $1,83 \%$ \\
\hline 18- Porto Velho & $40,36 \%$ & $56,56 \%$ & $12,76 \%$ & $21,44 \%$ & $3,28 \%$ & $5,30 \%$ & $0,61 \%$ & $1,16 \%$ \\
\hline 19- Recife & $41,24 \%$ & $54,21 \%$ & $11,24 \%$ & $18,87 \%$ & $2,72 \%$ & $4,91 \%$ & $0,63 \%$ & $1,41 \%$ \\
\hline 20- Rio Branco & $39,74 \%$ & $39,59 \%$ & $11,62 \%$ & $14,39 \%$ & $2,72 \%$ & $4,73 \%$ & $0,86 \%$ & $1,11 \%$ \\
\hline 21- Rio de Janeiro & $45,31 \%$ & $58,49 \%$ & $12,16 \%$ & $21,05 \%$ & $3,32 \%$ & $6,37 \%$ & $0,91 \%$ & $1,66 \%$ \\
\hline 22- Salvador & $37,34 \%$ & $49,27 \%$ & $9,21 \%$ & $16,96 \%$ & $1,61 \%$ & $4,93 \%$ & $0,55 \%$ & $1,51 \%$ \\
\hline 23- São Luís & $30,41 \%$ & $48,59 \%$ & $7,10 \%$ & $14,25 \%$ & $1,71 \%$ & $2,92 \%$ & $0,45 \%$ & $0,76 \%$ \\
\hline 24- São Paulo & $41,68 \%$ & $60,10 \%$ & $10,63 \%$ & $21,70 \%$ & $2,90 \%$ & $5,85 \%$ & $1,34 \%$ & $1,77 \%$ \\
\hline 25- Teresina & $32,23 \%$ & $47,90 \%$ & $8,21 \%$ & $15,72 \%$ & $1,60 \%$ & $3,95 \%$ & $0,40 \%$ & $1,20 \%$ \\
\hline 26- Vitória & $37,03 \%$ & $50,03 \%$ & $8,97 \%$ & $16,83 \%$ & $2,17 \%$ & $4,84 \%$ & $0,30 \%$ & $1,46 \%$ \\
\hline 27- Distrito Federal & $39,50 \%$ & $59,27 \%$ & $10,42 \%$ & $19,22 \%$ & $2,35 \%$ & $4,20 \%$ & $0,45 \%$ & $1,23 \%$ \\
\hline
\end{tabular}

Nota: ${ }^{a}$ foram desconsideradas as mulheres que manifestaram situação de gravidez na ocasião das entrevistas para o cálculo do IMC médio; ${ }^{b}$ parcela da população residente nas capitais do Brasil.

O aumento expressivo do IMC médio e da prevalência de obesidade nas populações das capitais do Brasil pode ser desencadeada por diversos fatores como aumento do sedentarismo e mudanças nos hábitos alimentares, aumento no consumo de alimentos processados e ultraprocessados ${ }^{19,20}$. Nesse sentido, a exemplo de outros países, iniciativas que visem regular o consumo de tais alimentos por parte da população, e regulamentar as ações das empresas do setor alimentício são estratégias que podem desempenhar um papel fundamental na redução do consumo de tais alimentos ${ }^{21-23}$, resultando em melhora nos indicadores de saúde e fatores de risco da população do Brasil.

O aumento da prevalência da obesidade na população brasileira tem implicações severas à saúde pública e à economia do país ${ }^{19,24,25}$. De fato, estudo anterior estimou que os custos totais de hipertensão, diabetes e obesidade ao SUS no ano de 2018 chegou a cerca de $R \$ 3,45$ bilhões, sendo $59 \%$ destinado ao tratamento da hipertensão, 30\% ao tratamento da diabetes e $11 \%$ ao da obesidade ${ }^{26}$. Logo, a prevalência das DCNT associadas à obesidade representa elevado custo ao sistema público de saúde do Brasil, e iniciativas tanto públicas como privadas de redução dos fatores de risco que potencializam o aumento de tais doenças são necessárias.
Observa-se que todas as capitais apresentam prevalências alarmantes de sobrepeso e obesidade, mas confere-se maior para Cuiabá, Porto Alegre, São Paulo, Distrito Federal e Rio de Janeiro, que apresentam as maiores parcelas da população em situação de sobrepeso e obesidade em seus três níveis. Por outro lado, Boa Vista, Macapá e Rio Branco apresentam pouco ou nenhum aumento nas prevalências de sobrepeso e obesidade em suas populações. De fato, a parcela da população em situação de sobrepeso em Boa Vista passou de 38,64\% em 2006 para $31,66 \%$ em 2018, e as parcelas da população em situação de obesidade (nos três níveis) permaneceu praticamente inalterada, embora se tenha observado aumento expressivo na prevalência de diabetes e dislipidemia (Tabela 1).

A Figura 1 é composta de oito diagramas de dispersão que analisam a relação entre a idade dos respondentes das pesquisas VIGITEL com o IMC médio (Fig. 1a), prevalência de obesidade I (Fig. 1b), prevalência de obesidade II (Fig. 1c), prevalência de diabetes (Fig. 1d), prevalência de pressão alta (Fig. 1e), prevalência de dislipidemia (Fig. 1f), prevalência de infarto, derrame e AVC (Fig. 1g), e autopercepção do estado geral de saúde (Fig. 1h). 

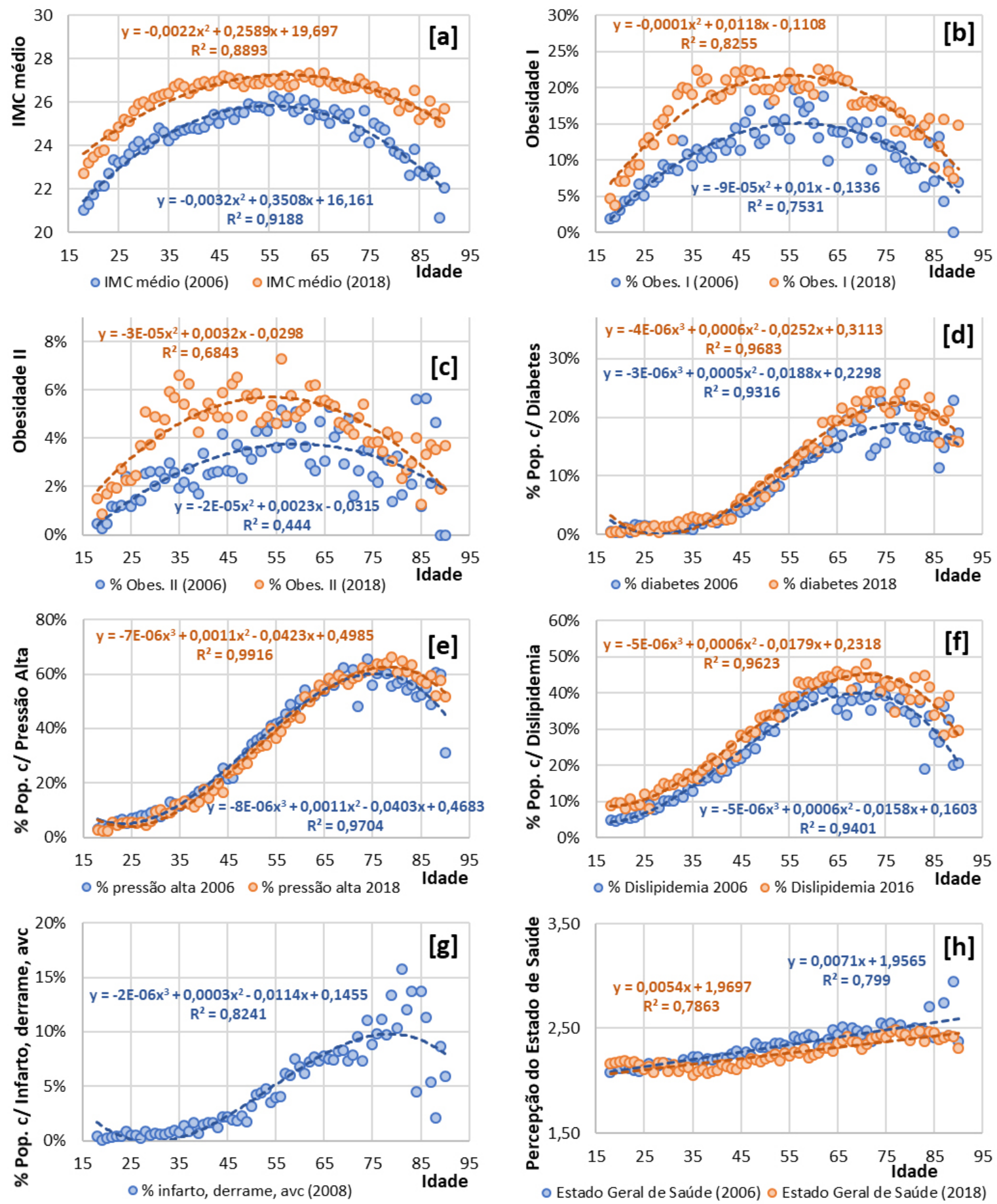

Nota: Para os gráficos [a], [b] e [c] foram excluídas as mulheres que manifestaram situação de gravidez na ocasião das entrevistas.

Figura 1: Relação entre idade e indicadores de saúde (IMC médio, prevalência dos níveis de obesidade I e II, e prevalências de diabetes, pressão alta e dislipidemia)

Os resultados mostram que o IMC médio da população aumentou de 2006 para 2018 proporcionalmente em todas as idades nas populações das capitais do Brasil, além de ser também observado que o IMC médio é maior na faixa etária entre 45 e 65 anos. A curva de tendência que melhor se ajusta aos dados é a equação de segundo grau com elevados valores de $R^{2}$ 
(Fig. 1a). Interpretação semelhante é dada para a análise da relação entre a idade e a prevalência populacional da obesidade I (Fig. 1b), uma vez que a equação de segundo grau apresenta ajuste satisfatório aos dados, e que a faixa etária com maior proporção populacional de obesos I é por volta dos 55 anos. A relação entre a idade e as prevalências de diabetes, pressão alta e dislipidemia mostra que a partir dos 40 anos a prevalência de tais comorbidades apresenta um acentuado crescimento, atingindo o seu pico na faixa entre 70 e 80 anos, sendo que a equação polinomial de terceiro grau apresenta melhor ajuste aos dados.

O envelhecimento tende a estar associado a um aumento da gordura corporal, uma vez que o aumento da idade leva a uma diminuição da secreção do hormônio de crescimento, diminuindo a taxa de metabolismo basal e a massa magra e aumentando a quantidade de gordura corpo$\mathrm{ral}^{27}$. Isso pode levar a um aumento significativo do IMC no decorrer do processo de envelhecimento. Ademais, evidências anteriores associam a variação e ganho de peso na vida adulta com o aumento do risco de mortalidade e de desenvolvimento de DCNT, relacionando ainda o ganho de peso em idade adulta com o aumento da incidência de alguns tipos de câncer e doença cardiovasculares $^{28-30}$. Também, estudos anteriores demonstram a significância existente ente o aumento da idade, além de outras características sociodemográficas, com o aumento do IMC, recomendando que estratégias de promoção de saúde pública relacionadas à prevenção do ganho de peso ao nível populacional devem considerar importantes fatores sociodemográficos ${ }^{31,32}$.

Seguindo a equação estimada para a prevalência de diabetes em 2018, o ápice verificado foi de aproximadamente $22 \%$ observado na faixa etária de 78 anos (Fig. 1d). Para a prevalência de pressão alta em 2018, o ápice verificado foi de aproximadamente $62 \%$ observado na faixa etária de 78 anos (Fig. 1e). Já para a prevalência de dislipidemia em 2018, o ápice verificado foi de aproximadamente $56 \%$ observado na faixa etária de 73 anos (Fig. 1f). No que se refere à prevalência de doenças cardiovasculares (infarto, derrame e AVC), cujos dados disponíveis são de 2008, o ápice verificado na equação estimada foi de aproximadamente $10 \%$ observado na faixa etária de 78 anos, embora os dados observáveis mostrem prevalência registrada de $16 \%$ aos 81 anos (Fig. $1 \mathrm{~g}$ ). Embora com o avançar da idade da população a prevalência das comorbidades aumente expressivamente, o aumento na autopercepção de piora do estado de saúde aumenta de forma muito pouco expressiva (Fig. 1h), sugerindo que a prevalência de diabetes, pressão alta e dislipidemia não impacta de forma direta na autopercepção de piora do estado geral de saúde das pessoas.

Evidência importante deste estudo é fraca conexão observada entre os indicadores de saúde e a autopercepção da população com relação à sua saúde, uma vez que os resultados observados para as variáveis que avaliam a saúde da população mostraram expressiva piora de 2006 a 2018, enquanto a variável relacionada à autopercepção de estado de saúde apresentou mudança inexpressiva, reforçando os achados do estudo que mostra que as pessoas tendem a não associar o peso à saúde ${ }^{33}$.

A Figura 2 complementa a análise anterior apresentando também oito diagramas de dispersão que objetivam analisar a relação entre a quantidade de anos de estudo dos respondentes com as variáveis relacionadas à saúde das populações das capitais do Brasil. 

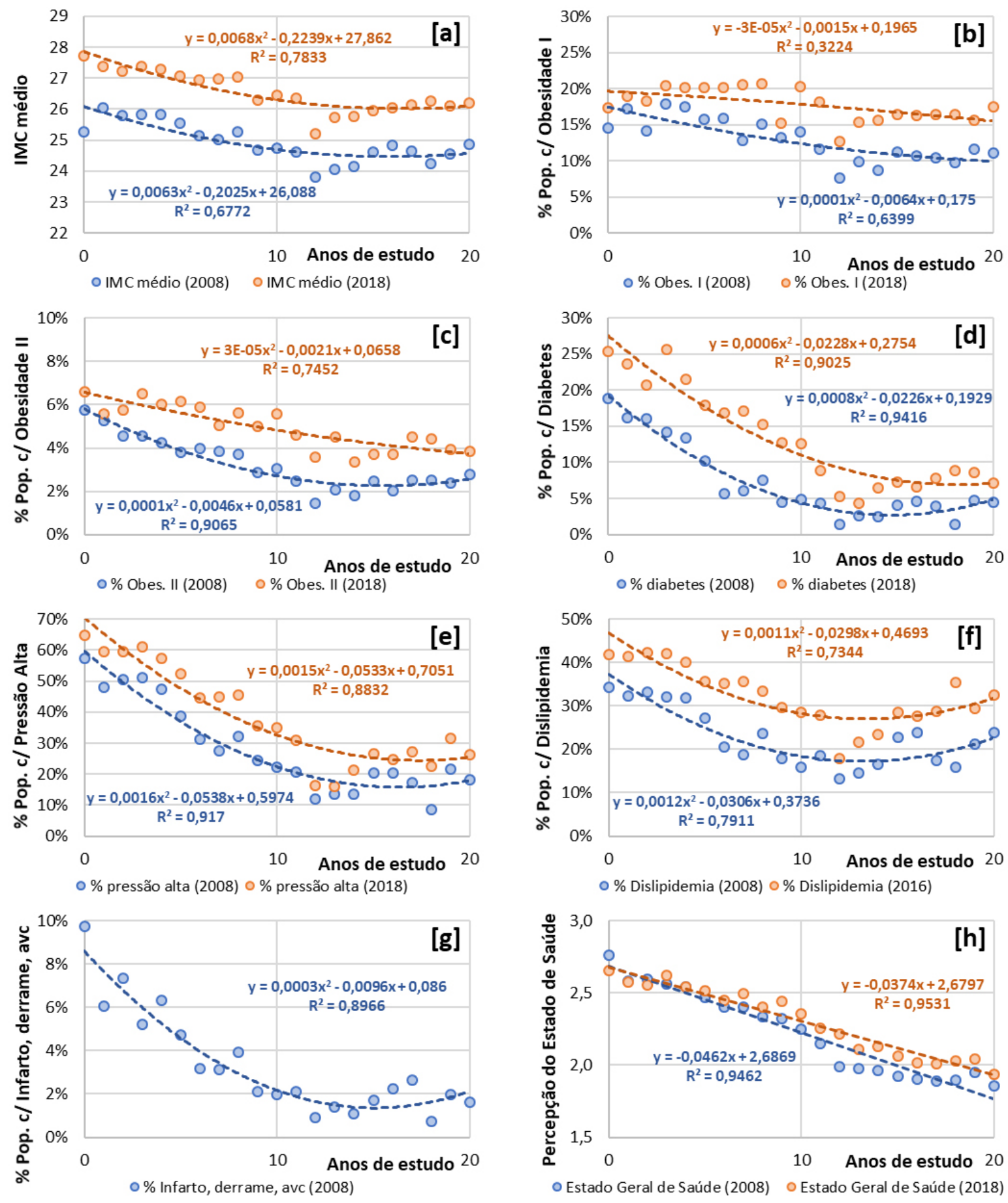

Nota: Para os gráficos [a], [b] e [c] foram excluídas as mulheres que manifestaram situação de gravidez na ocasião das entrevistas.

Figura 2: Relação entre anos de estudo e indicadores de saúde (IMC médio, prevalência dos níveis de obesidade I e II, e prevalências de diabetes, pressão alta e dislipidemia)

Os resultados mostram que o IMC médio da população tende a diminuir, tanto para os dados de 2006 quanto para os de 2018, conforme aumenta a quantidade de anos de estudo da população (Fig. 2a). Também é observado que o aumento na quantidade de anos de estudo da população tende a reduzir a prevalência da obesidade I (Fig. 2b), obesidade II (Fig. 2c), diabetes 
(Fig. 2d), pressão alta (Fig. 2e), dislipidemia (Fig. 2f), e doenças cardiovasculares (Fig. 2g). Ademais, a autopercepção dos respondentes é de uma saúde melhor conforme aumenta a quantidade de anos de estudo (Fig. 2f).

Outro achado de destaque deste estudo é a relação existente entre o número de anos de estudos e os indicadores de saúde, evidenciando que a educação formal está associada a um melhor quadro geral de saúde da população. Este resultado corrobora outros achados, como estudo conduzido no Canadá que evidenciou que indivíduos com menos anos de educação formal consomem mais alimentos ultraprocessados, e que tal consumo está associado positivamente à obesidade ${ }^{34}$. Seguindo outra linha de investigação, estudo anterior evidenciou que o nível de educação e intervenção dos pais na alimentação das crianças tende a desencadear hábitos alimentares mais saudáveis ${ }^{35}$. Logo, é razoável assumir que um incremento no nível educacional de uma população tem potencial elevado de promover melhoria no quadro de saúde pública, reduzindo potencialmente os gastos em saúde e melhorando a qualidade de vida populacional.

\section{CONCLUSÃO}

Este estudo evidencia que o IMC médio populacional está aumentando de forma acentuada, e que as prevalências de DCNT, principalmente diabetes e hipertensão, acompanham tal crescimento. É razoável assumir que tal agravamento do quadro de saúde das populações das capitais do Brasil tem potencial de elevar drasticamente nos próximos anos as demandas da população por serviços do sistema de saúde do Brasil, o que desencadeia a necessidade de aumentar a capacidade e destinar maior montante de recurso financeiro ao sistema de saúde, tanto público como privado.

Também foi evidenciado que o IMC médio da população é maior na faixa etária entre 45 e 65 anos, e as prevalências de diabetes, pressão alta e dislipidemia apresentam um crescimento acentuado partir dos 40 anos, atingindo o ápice na faixa etária entre 70 e 80 anos. 0 estudo também evidenciou que o nível de educação formal, avaliado por meio da quantidade de anos de estudo, possui relação acentuada com as prevalências de obesidade e DCNT ao passo que, quanto mais anos de estudos possui a população, menor são as prevalências de tais comorbidades. Dessa forma, iniciativas, tanto públicas como privadas, de redução dos fatores de risco que potencializam o aumento da obesidade e de DCNT são necessárias. Ademais, o aumento do nível educacional de uma população tem potencial de promover melhoria significativa no quadro de saúde pública, reduzindo os gastos em saúde e melhorando a qualidade de vida populacional.

Este estudo possui limitações importantes uma vez que utiliza apenas dados das edições do VIGITEL. Com isso, os dados analisados são de indivíduos residentes apenas nas capitais dos estados do Brasil, o que impede uma análise das populações residentes em municípios do interior ou da área rural, que representa grande parcela da população brasileira. Com isso, sugere-se que sejam conduzidos estudos adicionais que objetivem analisar e monitorar a situação de obesidade e sobrepeso na população brasileira residente em municípios do interior e da zona rural, além de identificar os principais fatores que desencadeiam a possível prevalência de obesidade nesses locais. Sugere-se também que sejam conduzidos estudos adicionais que visem analisar as repercussões do agravamento da obesidade e das DCNT nos custos associados ao SUS, assim como outros custos à sociedade, como implicações sociais e implicações trabalhistas.

Oportunamente, este estudo recomenda que sejam delineadas e adotadas iniciativas públicas mais efetivas no sentido de promover uma redução significativa na prevalência de doenças não transmissíveis no Brasil, principalmente a promoção de políticas públicas direcionadas a crianças e adolescentes.

\section{REFERÊNCIAS}

1. Abbade EB, Dewes H. Behavioral and societal drivers of an obesogenic environment worldwide. Nutr Food Sci 2015; 45: 229-241.

2. Dias PC, Henriques P, Anjos LA dos, et al. Obesidade e políticas públicas: concepções e estratégias adotadas pelo governo brasileiro. Cad Saúde Pública 2017; 33: e00006016.

3. Giskes $\mathrm{K}$, van Lenthe $\mathrm{F}$, Avendano-Pabon M, et al. A systematic review of environmental factors and obesogenic dietary intakes among adults: Are we getting closer to understanding obesogenic environments? Obes Rev 2011; 12: e95-e106.

4. Rech DC, Borfe L, Emmanouilidis A, et al. As políticas públicas e o enfrentamento da obesidade no Brasil: uma 
revisão reflexiva. Rev Epidemiol E Controle Infecção 2016; 1: 192-202.

5. OMS [Organização Mundial da Saúde]. Global Health Observatory (GHO), http://www.who.int/gho/database/ en/ (2018, accessed 5 August 2018).

6. MS [Ministério da Saúde]. Vigilância de Fatores de Risco e Proteção para Doenças Crônicas por Inquérito Telefônico, Vigitel 2017. Ministério da Saúde Brasília, http://bvsms.saude.gov.br/bvs/publicacoes/vigitel_brasil_2017_vigilancia_fatores_riscos.pdf (2017).

7. McTigue K, Larson JC, Valoski A, et al. Mortality and cardiac and vascular outcomes in extremely obese women. J Am Med Assoc 2006; 296: 79-86.

8. Misra A, Khurana L. Obesity and the metabolic syndrome in developing countries. J Clin Endocrinol Metab 2008; 93: s9-s30.

9. Moghaddam AA, Woodward M, Huxley R. Obesity and risk of colorectal cancer: A meta-analysis of 31 studies with 70,000 events. Cancer Epidemiol Biomarkers Prev 2007; 16: 2533-2547.

10. Abbade EB. The relationships between obesity-increasing risk factors for public health, environmental impacts, and health expenditures worldwide. Manag Environ Qual Int J 2018; 29: 131-147.

11. Buchmueller TC, Johar M. Obesity and health expenditures: Evidence from Australia. Econ Hum Biol 2015; 17: 42-58.

12. Specchia ML, Veneziano MA, Cadeddu C, et al. Economic impact of adult obesity on health systems: A systematic review. Eur J Public Health 2015; 25: 255-262.

13. Spieker EA, Pyzocha N. Economic Impact of Obesity. Prim Care - Clin Off Pract 2016; 43: 83-95.

14. Abbade EB. Análise das internações hospitalares para procedimentos de cirurgias bariátricas financiadas pelo SUS em âmbito nacional. Med Ribeirao Preto Online 2019; 52: 201-211.

15. WHO. The top 10 causes of death, https://www.who int/news-room/fact-sheets/detail/the-top-10-causes-of-death (2018, accessed 10 August 2020).

16. WHO. Noncommunicable diseases country profiles 2018 https://apps.who.int/iris/handle/10665/274512 (2018, accessed 4 September 2020).

17. MS [Ministério da Saúde]. Vigilância de fatores de risco e protecão para doenças crônicas por inquérito telefônico: VIGITEL 2011. Brasília: Ministério da Saúde, 2012.

18. World Health Organization. Physical status: The use of and interpretation of anthropometry, Report of a WHO Expert Committee. Geneva: World Health Organization, https://apps.who.int/iris/bitstream/handle/10665/37003/WHO_TRS_854.pdf (1995, accessed 5 May 2020).

19. Canella DS, Levy RB, Martins APB, et al. Ultra-Processed Food Products and Obesity in Brazilian Households (2008-2009). PLOS ONE 2014; 9: e92752.

20. Cunha DB, Costa THM da, Veiga GV da, et al. Ultraprocessed food consumption and adiposity trajectories in a Brazilian cohort of adolescents: ELANA study. Nutr Diabetes 2018; 8: 1-9.

21. Castronuovo L, Allemandi L, Tiscornia V, et al. Analysis of a voluntary initiative to reduce sodium in processed and ultra-processed food products in Argentina: the views of public and private sector representatives. Cad Saude Publica 2017; 33: e00014316.

22. Kanter R, Reyes M, Swinburn B, et al. The food supply prior to the implementation of the Chilean Law of Food Labeling and Advertising. Nutrients 2019; 11: 52.

23. Nilson EA, Spaniol AM, Gonçalves VS, et al. Sodium reduction in processed foods in Brazil: analysis of food categories and voluntary targets from 2011 to 2017. Nutrients 2017; 9: 742.

24. Dee A, Kearns K, O'Neill C, et al. The direct and indirect costs of both overweight and obesity: a systematic review. BMC Res Notes 2014; 7: 242.

25. Malta DC, Andrade SC, Claro RM, et al. Trends in prevalence of overweight and obesity in adults in 26 Brazilian state capitals and the Federal District from 2006 to 2012. Rev Bras Epidemiol 2014; 17: 267-276.

26. Nilson EAF, Andrade $R$ da CS, Brito DA de, et al. Custos atribuíveis a obesidade, hipertensão e diabetes no Sistema Unico de Saúde, Brasil, 2018. Rev Panam Salud Pública 2020; 44: e32.

27. Rudman D, Feller AG, Nagraj HS, et al. Effects of human growth hormone in men over 60 years old. $N$ Engl J Med 1990; 323: 1-6.

28. WHO. Obesity: preventing and managing the global epidemic. World Health Organization, 2000.

29. WHO. Diet, nutrition, and the prevention of chronic diseases: report of a joint WHO/FAO expert consultation. World Health Organization, 2003.

30. Vergnaud A-C, Bertrais S, Oppert J-M, et al. Weight fluctuations and risk for metabolic syndrome in an adult cohort. Int J Obes 2008; 32: 315-321.

31. Coelho MSPH, Assis MAA de, Moura EC. Body mass index increase after the age of 20 and associations with risk or protection factors for chronic non-communicable diseases. Arq Bras Endocrinol Amp Metabol 2009; 53: 1146-1156.

32. Van Lenthe FJ, Droomers M, Schrijvers CTM et al. Sociodemographic variables and 6 year change in body mass index: longitudinal results from the GLOBE study. Int J Obes 2000; 24: 1077-1084.

33. Pavão ALB, Werneck GL, Campos MR. Autoavaliação do estado de saúde e a associação com fatores sociodemográficos, hábitos de vida e morbidade na população: um inquérito nacional. Cad Saúde Pública 2013; 29: 723-734.

34. Nardocci M, Leclerc B-S, Louzada M-L, et al. Consumption of ultra-processed foods and obesity in Canada. Can J Public Health 2019; 110: 4-14.

35. Lwin MO, Shin W, Yee AZH, et al. A Parental Health Education Model of Children's Food Consumption: Influence on Children's Attitudes, Intention, and Consumption of Healthy and Unhealthy Foods. J Health Commun 2017; 22: 403-412. 


\title{
Agradecimentos
}

Esta pesquisa faz parte do projeto "Histórico e Projeção do Impacto Econômico da Obesidade e suas Consequentes Doenças Crônicas Não Transmissíveis (DCNT) no Sistema Único de Saúde" financiado pelo CNPq (Conselho Nacional de Desenvolvimento Científico e Tecnológico) (Processo no 442619/2019-7). $\mathrm{O}$ autor gostaria de agradecer ao CNPq pelo apoio financeiro concedido.

\section{Financiamento}

Este estudo foi financiado pelo CNPq (Conselho Nacional de Desenvolvimento Científico e Tecnológico) (Processo no 442619/2019-7).

\author{
Autor correspondente: \\ Eduardo Botti Abbade \\ eduardo.abbade@ufsm.br | edabbade@hotmail.com \\ Editor: \\ Prof. Dr Felipe Villela Gomes \\ Recebido em: 24/06/2020 \\ Aprovado em: 09/09/2020 \\ (c) Este é um artigo publicado em acesso aberto (Open Access) sob a licença Creative

